\title{
Sensing as a Service: Vision, Practices and Architecture
}

\author{
Maha Arooj \\ Department of Computer Science and IT, University of Lahore, Pakistan \\ Email: maha.arooj@cs.uol.edu.pk \\ Muhammad Asif \\ Department of Computer Science and IT, University of Lahore, Pakistan \\ Email: muhammad.asif@cs.uol.edu.pk
}

Received: 11 September 2018; Accepted: 20 June 2019; Published: 08 November 2019

\begin{abstract}
The Internet of Things (IoT) is becoming pervasive and immersive due to the recent advancements in communication and sensing technologies. The proliferation of smart devices and their sensing capabilities has opened new opportunities and business models. The billions of connected sensing devices are generating enormous amount of data. The sensing as a service concept has the potential to provide a wide variety of services to citizens, companies and public administrations. This paper presents a sensing as service vision for IoT in different domains such as agriculture, waste management, supply chain, traffic management and others. Moreover, different applications of sensing as service model is analyzed and discussed in detail. In this paper, we specifically propose a service oriented sensing as service architecture to realize the vision of sensing as a service. The proposed service oriented architecture has the potential to address the challenges of heterogeneity, integration and interoperability of a sensing as service concept and can open new business opportunities.
\end{abstract}

Index Terms-Smart city, Cyber-physical systems, Ubiquitous sensing, Smart environment, IoT Security

\section{INTRODUCTION}

Recent revolutions in wireless communications, embedded application development platforms, sensing technologies and cloud computing are driving networked objects and services towards a new computing and communication paradigm, commonly referred to as the Internet of Things (IoT). IoT paradigm visualizes the points of interests as 'things'. The term 'thing' virtually encircles every object that surrounds us, ranging from information sources (sensors, identifiers etc.) to impellers (actuators etc.). The basic idea is to connect these things to the larger internet. This integration will result in generation of enormous amounts of data. If this data is subjected to rich contextual information and sophisticated analytics, this can yield insights to information, which could not have been possible to achieve otherwise. IoT create a large influence on technological market using 18 billion sensor devices as predicted by 2022. It is estimated that many IoT services will offer worldwide influence across millions of tiny devices. [1]

It is one of the most vital architectures for several capable applications such as smart healthcare, smart environment, intelligent transport, home automation etc. [2]. Therefore, IoT can transform our personal, social, societal and economic aspects of life by adding new dimensions to how people and things can communicate and collaborate. Industrial IoT is also an emerging area which consists of wide diversity of sensors, actuators, and controllers [4]. The sensed data from this variety of sensing devices has opened an era of big data analytics in IoT.

Sensing as a Service $\left(\mathrm{S}^{2} \mathrm{aaS}\right)$ model is a vision of a corporate model that indorses data trading between sensor owners and data consumers. This $\left(\mathrm{S}^{2} \mathrm{aaS}\right)$ model enables bringing the sensors under the umbrella of shared sensing infrastructure for everyone. Data is acquired for publishing from these sensors for fee, or for other valueadded services. Enabling such infrastructure can address the problem of having high concentration of distributed sensing capabilities. This paper provides state of the art in sensing as a service and more specifically provides extended view of Sensing as a Service Model in a variety of domains and developed architecture for $S^{2}$ aaS. Security of IoT based systems and applications is a critical element. However, due to brevity and research in progress kept this aspect for future research.

The rest of the article is organized as follows: Section II presents the vision of sensing as a service concept. Section III presents an overview of the applications and practices of the sensing as a service concept. Sensing as a service $\left(S^{2}\right.$ aaS) architecture is presented in section IV and section $\mathrm{V}$ concludes the paper.

\section{SENSING AS A SERVICE:VISION}

The advancements in sensor technologies are providing ubiquitous sensing platforms which are core for any IoT solutions. Sensors can detect and observe changes in positions, humidity, temperature, light and other various 
environmental parameters. The deployment of sensing environment is increasing due to the advancement in sensor technology and reduction in production and processing cost of sensors. There is significant growth in investment for sensor technology. According to [7] the number of investing companies shows growth of 3\%, and top performers investment increase by $7 \%$ and $54 \%$ of top performers said about increase in investment of sensor technology. Moreover, according to PwC's 6th Annual Digital IQ survey of nearly 1,500 business and technology executives' investment in IoT is underway.

IoT emerge the concept of 'smart city' globally, where it enriched the infrastructure monitoring, public security, Smart Transport Surveillance and Intelligent Optimization systems are being installed on a city-wide scale. The industries such as power and energy, automotive, healthcare, retail, and entertainment are investing in IoT sensor technology. [6] The growth in pervasive diffusion of sensors to people, places, processes and products to sense, gather and analyze data is augmenting intelligence for decision making. A wave of organic smart labels promises a dramatically cost reduction to provide on-label sensor which are becoming popular in smart supply chain, healthcare and remote monitoring [3]. According to Cluster of European research projects on the IoT [42], things are becoming active participants in business, information and social process that can sense and share information about real/physical world events. $\mathrm{S}^{2}$ aaS vision is looking to exploit the sensing opportunities offered by IoT and open new opportunities and business models. A new paradigm of Internet of Everything (IoE) is emerging which includes people, processes, things and data. A variety of other different types of Internet of Things such as Internet of Nano Things (IoNT), Internet of Mission-critical Things (IoMCT) and Internet of Mobile Things (IoMT) are elaborate in [].

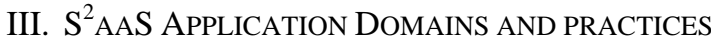

We presented four important domains which we believe can help to understand the vision and concept of Sensing as service: smart agriculture, supply chain management, and smart traffic. In this section, we briefly discuss the relevance and potential impact of sensing as a service vision in the following application domains as illustrated in Fig 1.

\section{A. Agriculture}

Agriculture is the most essential part of world's economy and facilitates many business entities and communities around the world. According to World Economic Forum [7], the demand of feed of 9 billion world population will rise to $70 \%$ by 2050 . Grow Africa initiative [8]is a kind of joint venture by World Economic Forum and African Union Commission (AUC) to target collaboration among key stakeholders and improve agricultural growth. To achieve the objective of growth rate by $20 \%$ requires a transformation of the agriculture sector by increasing the collaboration by all stakeholders including farmers, marketers, government, civil society, and private sector. Smart agriculture systems can pave the way to achieve the global objectives of food security and agriculture. Smart agriculture system mostly defined in five factors: environmental, social, economic, geographical and technical aspects. Smart agriculture systems have the potential in provisioning in agricultural management, production and overall distribution processes $[9,10]$.

The enabling technologies in IoT are going to modernize agriculture around the world particularly in developed countries [5, 11]. The smart agriculture systems are widely being used in crop breeding, preservation of crops, forestry and insect operation, and agricultural meteorological conditions [11, 12]. Sensing capabilities and platforms are required to monitor the plant growth status, soil conditions, pest controlling, and weather conditions. Different open IoT platform initiatives such as OpenIoT [13] and Phenonet [14] are being utilized to develop sensing and publishing platforms for smart agriculture.

IoT in agriculture has opened new ways of cultivating soil for farmers and growers with the use of cheap and easy to deploy sensors. Different studies [11, 15] are exploiting various kinds of sensors and enabling technologies to sense data for farmers, researchers and consumers. The Sensing as Service can be an ideal case of application for smart agriculture. We have briefly described the application of $S^{2}$ aaS to deliver the sensing as a service for smart agriculture.

Sensors and Sensor Owner Sensors can be deployed by farmers or landlords, research organizations or institutions and can be considered as a sensor owner. Sensors can detect and measure humidity, climate condition, crop production, temperature, and pest control sensing. Sensing can utilize multiple sensors that can be attached to an entity or a device. The sensors and actuators can be used to specifically control the activity for example the meditation of fertilizer in territory that rely on the material assembled from the soil itself, the climate temperature, and other environmental factors. Sensor Owner can decide to give permission to publish the sensors or not. The owner needs to be registered with sensor publisher to grant access and agreement to the sensor publishers to publish the sensors and data as shown in Fig 2.

Sensor publisher can detect the sensors, interact with sensor owners and can provide requested services to the consumers. The sensor publisher (SP) will identify the available sensors, assembles the service about the sensor availability, owner inclinations and constraints, agreements, predictable return etc. After the registration, sensor publisher waits until a sensor consumer sends the request. When SP receives any request it is forwarded to sensor owner to acceptor discard. SP executes a business process for sensor owners and data consumers.

Extended Service Provider can be considered as an intelligent layer of the model. This layer helps the consumers to communicate with SP and can provide semantically enriched data to make it explicit for 
consumers. For example, scientists want to examine the soil and crop status according to climate condition in some region and can request the specific data which must be pragmatic for the task.

Sensor Data Consumers: The data consumers can be the government agencies, microbiologist, agriculturist, farmers, research organizations, fertilizer and pesticides companies. The consumers can be registered with domain specific ESPs to acquire the required data or analytics depending on the nature of the requirement.

\section{B. Supply Chain Management}

Supply chain management (SCM) is a set of coordinated activities for incorporating the dealers, producers, transporters, and consumers conveniently so that the right item or service can be delivered [16]. Global supply chain forum has identified eight main process of SCM: consumer relationship and service management, demand management, order fulfillment, manufacturing, flow management, procurement, product development and commercialization and return [17-19]. Supply chain particularly in food (fresh or processed) requires extra measures to manage the quality parameter of food items and maintain the food degradation process. Long supply chains of fresh or unpreserved food stuff can be affected by extraordinary hazardous elements [20-24]. The operational efficiency in fleet management, cargo integrity monitoring, and storage condition control, optimization of warehouse workload, inventory tracking and analytics can be achieved with IoT technology In the following, we have briefly described the layers of sensing as a service model in smart supply chain domain.

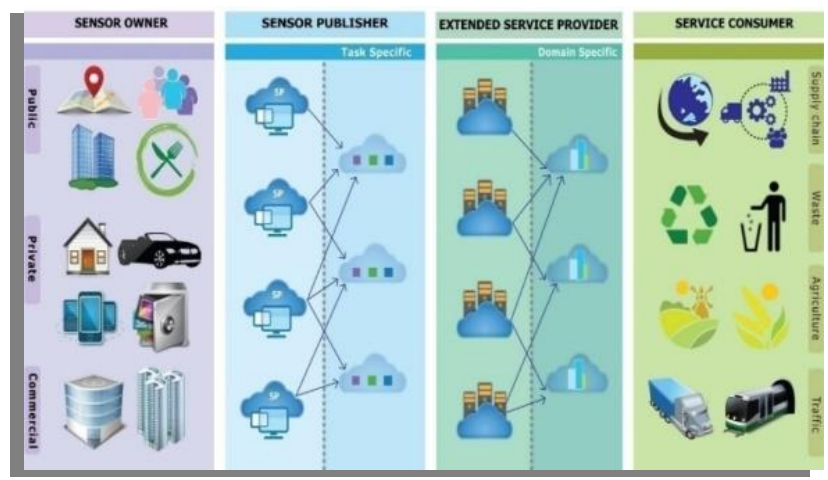

Fig.1. Extended Sensing as a Service Model

Sensor owner: In supply chain and logistics ecosystem, many players can take part in sensing activity and can produce rich data. In this case, sensor owners can be suppliers, manufacturers, warehouses, distribution centers, storages, and cargos. Supplier can deploy sensors in supplier management firm or in vehicles that transfer that raw material to the manufacturers. Sensor owner can also be some third parties or systems. Transport vehicles have the internal sensors to monitor the freshness and safety parameter of food material throughout the travel time. Environmental condition can be depending on time of travel, temperature, humidity level, carbon quantity in atmosphere etc.
Sensor Publisher: The sensor publisher has access to the sensors registered by the owners to take part in sensing activity. The sensors publisher can make the sensors available to different ESPs depending on the nature of the sensing task. The sensing task may include gathering data about fleets in transition, environmental conditions of the operating environment or traffic conditions.

Extended service provider can be domain specific depending of the nature of the service requirement. For example, a traffic monitoring ESP can provide rich data required for fleet management. All the involved stakeholders can fetch and process sensor data in real time in order to accomplish their own objective. Similarly, manufacturer may use sensor data to monitor the quality of product in terms of temperature, environment and time consumption. ESPs can provide data services and intelligence required by different stakeholders in a supply chain ecosystem.

Sensor data consumer: The sensor data consumers in supply chain ecosystem can be manufacturers, suppliers, warehouse owners, fleet management, and end users. The consumers do not need to communicate with sensors and sensor owner directly ESP and SP will bridge the data requirements. For example, a customer deploys sensors in his home refrigerator. After purchasing the product these sensors sensed the quality of purchase item according to that environment and then it allows giving feedback to the manufacturer or supplier company about the purity and maintainability time of the item.

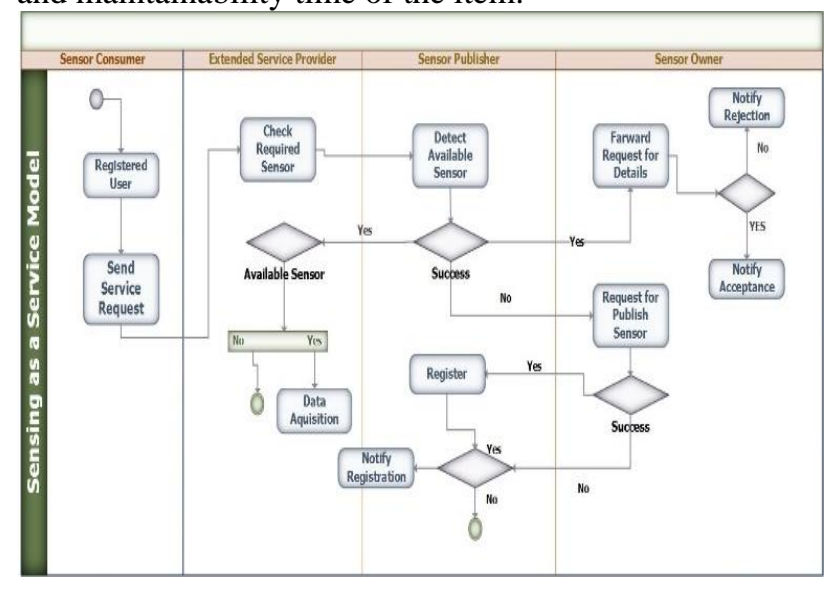

Fig. 2. Activity Diagram of $S^{2}$ aaS Model

\section{Traffic Management}

Smart traffic monitoring and management is a growing need of smart cities. With the enabling technologies, now it is becoming possible to have a smart infrastructure to control the vehicles flow on road, provide efficient and safe road journey, accident handling, help in preventing the traffic congestion, save the travel time or cost and fuel consumption [25].The objectives of smart traffic monitoring can be achieved by collecting real time data and observation of different traffic conditions.

Intelligent traffic management system according to $\mathrm{S}^{2}$ aaS model can have Sensor Owners which can be vehicle drivers, traffic police, and road governing 
authorities. They can deploy sensors in the vehicles, engines or in police base station. The sensors can detect the traffic flow conditions, sense vehicle speed, identify locations, alert from emergency situation on road etc. Multiple sensors can be attached to different traffic monitoring objects to detect different events. The sensors can sense the data and upload it to the cloud platform for further processing and transmission.

Sensor Publisher can detect the availability of sensors from sensor owners. For example, in an emergency scenario, a smart ambulance can identify the location of accident and can take the victim to the nearest hospital safely and timely [26]. The SP can detect the sensor presence on the road and get permission to publish the sensor data for ambulance guidance. SP's can provide services to all participants in the scenarios and can allow particular sensors to retrieve information about sensed data, user requirement and their boundaries. However, SPs can provide data to deliver different services to the all kinds of participants of smart traffic.

Extended Service Provider in intelligent traffic can provide different traffic management services to the different data consumers which can be individuals, any public or commercial organization. For example, alert applications gives info about any alarming situation on road and also some applications give the required information to user as they required regarding to their journey. This layer clarify the data assembled about all services retrieve by user e.g. alert condition, road condition etc. from publisher cloud platform and make available a supportive interface between SP's and Consumers. This data acquisition contains precise and particular semantics of all data to make it explicit for every consumer according to the situation.

Sensor Data Consumer Layer Different user (individuals, public or commercial organization) have to be registered with particular ESPs to consume the sensed data. It doesn't communicate with other sensors and sensor owner directly involve domain specific ESPs to disseminate the data and analytics. User may be drivers, city governance, vehicular manufacturing company, and traffic patrolling police etc. can get different value added services related to smart traffic.

\section{SENSING AS A SERVICE (S ${ }^{2}$ AAS) ArChitecture}

Service-oriented computing is gaining acknowledgement as a central paradigm for IoT Systems and is already accepted as a well-founded reference paradigm for Internet computing [27, 28]. Services are self-descriptive, platform agonistic computational elements and can be anything from simple requests to complicated business process [27]. To meet the requirements in a heterogeneous environment of IoT, the services should be technology neutral, loosely coupled and support location transparency [27]. Due to the decentralized and heterogeneous nature of IoT, SOA based approaches seems feasible solutions to achieve integration and interoperability among the heterogeneous devices and services [29, 30]. The key design requirements for $\mathrm{S}^{2}$ aaS architectures are scalability, modularity, extensibility, and interoperability among heterogeneous things, services, and applications. In this paper, an architecture for sensing as service model using Service-oriented computing paradigm is presented to meet the challenges of sensor identification and discovery, sensor access and control and sensor data consumption. Previously, SOA has been applied to create multilayer architectures for IoT [29, 31-34]. SOA is widely used [35-38] in developing middleware technology and WSN due to its inherited features of abstraction, integration and interoperability. According to the International Telecommunication Union (ITU), the IoT architecture should consists of five layers: sensing, accessing, networking, middleware and application layer. In $S^{2}$ aaS model, the sensing task is treated as a service which is divided and delivered using a layered architecture shown in Fig 3. The conceptual layers of the architecture followed the $S^{2}$ aaS model: Sensor owner, Sensor Publisher, Extended Service Provider, and Sensor Consumer is presented.

In this section, we briefly describe the overall architecture (Fig. 3) and the functionality of each layer to deliver the Sensing as a service. The four layers of the sensing as a service architecture, their roles and interactions is presented as follows:

\section{A. Sensor Owner Layer}

With the increasing capability of identification and sensing technology, it is becoming possible to automatically sense and exchange information. This layer consists of sensors/things owned by same or different owners which can be used to provide data. This layer represents two major perspectives: the smart things and their ownership. The things having certain sensors at this layer can participate in sensing activity with the consent/agreement of the sensor owner. The things that are participating can be categorized according to their ownership as shown in Fig 3. The basic categorization of the things is: personal, public and commercial. The personal things may include household items such smart refrigerator, Smart TV, Game consoles, microwave oven, personal weather station, or any other things that has the capability to sense and communicate. The public sensing things may include parks, roads, or other infrastructure meant for sensing. There can be public or private organization that can take part in sensing activity. The third category is commercial sensors, are the organization that can deploy sensors at private, public places according to agreed policies.

This layer communicates with the Sensor Publisher layer to perform various tasks. The first task is to register a sensing thing for participating in the activity and make it available to the sensor publisher. The owner will decide to publish his/her things to take part in sensing activity. The tasks that can be performed at sensor owner layer may include:

- The sensor owner has to register with a particular SP or the multiple SPs to which he/she want to 
publish the sensing things. It requires a mechanism (application/service/system) to facilitate the registration process with certain SPs.

- The sensor owner has to make the sensing things discoverable and publishable to different SPs. The SPs and sensor owner should have mechanism that can help to register/unregister the sensing things according to the certain agreed policy and security parameters.

- The sensor owner has to define and execute a policy to manage restriction and conditions to access the things and return offers.

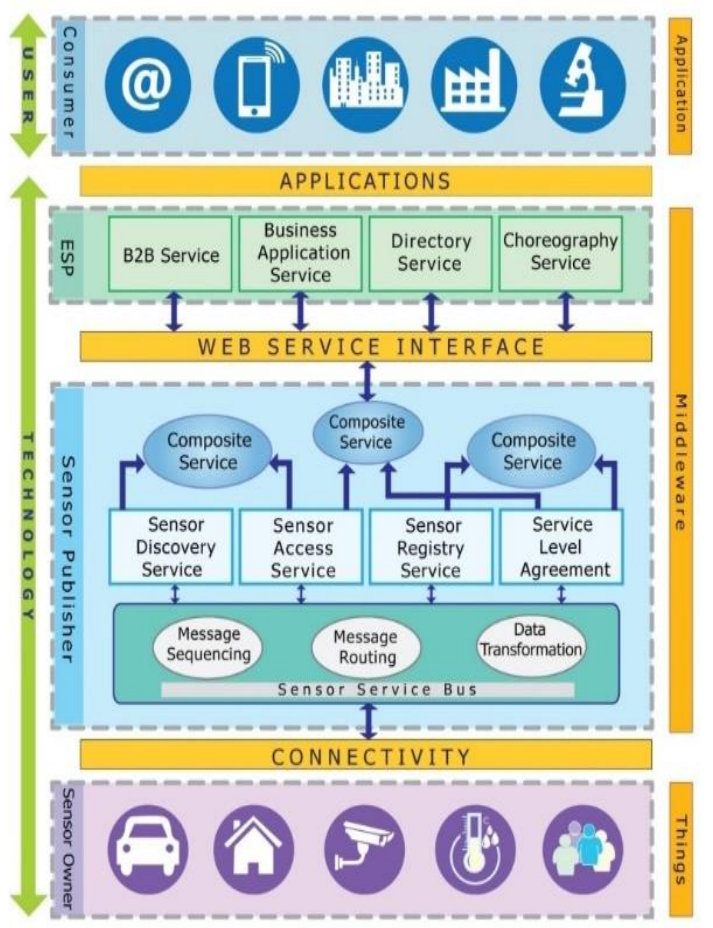

Fig.3. S ${ }^{2}$ aaS Architecture

\section{B. Sensor Publisher Layer}

This layer represents sensor publisher that can be used to detect available sensors, communicate with sensor owners, and get permissions to publish the sensors. Sensor publisher layer consists of modules or elements required for sensing task such as: Sensor Discovery Service, Sensor Access Service, Sensor Registry Service, and a Service level agreement. Sensor publishers are usually separate entities that can perform following tasks with sensor owner layer:

- Detect and communicate with available sensors from registered sensor owners.

- Get permissions and collect information about certain conditions (preferences, restriction, and expected return) to publish the sensor things.

Sensor publishers can be task specific and can take part to perform a common task required by different ESPs. Sensor publisher may require to sense from a set of heterogeneous devices and it becomes a challenging task to design a communication layer between Sensor owner and sensor publisher layer. Such kind of heterogeneity may create difficulties to achieve the requirements of communication layer such as dynamic, loose coupling, robustness, versatility, and flexibility [33]. A light weight sensor service bus is introduced to fulfill the need of sensor identification and discovery, sensor access and control. The light weight sensor service bus is central to the functioning of Sensor Publisher (SP) layer. Using a message bus model the infrastructure of sensor owners can be connected with extended service provider layer to provide end user services. The policies for access control and privacy regulations can be deployed using the sensor service bus. The sensor service bus is comparable to those used in main stream SOA architectures. Service may themselves be composed of other services depending on the requirements from extended service provider. Services may form a part of other services in an automated composition that should be adaptive and selfmanaging. The automated composition of services can provide advantages such as information typing, service optimization, functional redundancy and substitution and runtime optimization [28, 39].

\section{Extended Service Provider Layer}

This layer also relies on middleware technology that can provide seamlessly integrated services to the consumers. The ESP layer handles the interaction with the users or with the sensor provider layer. The layer listens to the requirements of consumers, translate, compose and forward the request to the sensor publisher. This also allows the consumers to invoke the web services, provide independent access to business process and services exposed. The layer communicates with sensor publisher layer via web interface to get information about available sensors from SPs to fulfill the consumers' requirements. This layer can provide domain specific ESPs to make the process easy and robust. The consumers may register with the respective ESPs to get the service. This layer can perform certain roles as depicted in Fig 3 such as B2B Service and directory services, service composition, service provisioning and management. The service delivery process at this layer also involves QoS management and control depending on users/applications' requirement. ESP layer will manage information exchange and storage, and will provide services and required information in an efficient way. A main role of the layer is to enable interaction and communication with the diverse kind of consumers. Web services choreography support action tags that state request and response and call tags demands external services. Service choreography covers combined procedures containing multiple services and their communications like the behavioral interface captures the manner of a single service that contributes in choreography [40-41].The layer can create, compose and schedule more suitable services to meet the requirement of the users.

\section{Sensor Consumer Layer}


This layer consists of different sensor data consumers that can be a person or an organization (public/private/commercial). The consumers can be of different kinds with different data needs to fulfill their purpose. The data consumer can register with different ESPs according to their needs and requirements. The consumers in our case are supply chain management, waste management, smart agriculture, and traffic domains. Sensor data consumers are required to be registered and communicate with the ESPs but not directly with sensor owner layer. Sensor data consumers can be novice or can have all the technical capabilities to select ESPs. Sensor consumer may register their interests/requirements with particular domain specific ESPs. However, we posit that domain specific ESPs can reduce the effort of finding appropriate ESPs for sensor data consumers. Similarly ESPs may need not to combine SPs for a particular task instead task specific SPs can be made available for ESPs. It is suggested that [5] sensor consumers with less technical capabilities should acquire sensor data through ESPs where complex task of selecting and combining the sensor is performed.

\section{CONCLUSION}

This paper presented a comprehensive overview of Sensing as a Service model and its applications in different domains such as Waste management, Agriculture, Supply Chain Management. The article introduced generic SOA based sensing as a service architecture. The main objective of the S2aaS architecture is to provide an infrastructure where sensing as a service concept can be realized and new business models of IoT can be achieved. The proposed architecture has the potential to provide scalability, modularity, extensibility and interoperability among the heterogeneous entities participating in sensing as a service paradigm. The future work will focus on developing different architectural elements and produce a proof of concept for sensing as service model. Moreover, security aspects of sensing as service model will also be implemented.

\section{REFERENCES}

[1] Novo, O., Blockchain Meets IoT: an Architecture for Scalable Access Management in IoT. IEEE Internet of Things Journal, 2018.

[2] Zhang, Y., et al., Smart Contract-Based Access Control for the Internet of Things. arXiv preprint arXiv:1802.04410, 2018.

[3] Kavis, M. The Smart Labels that will power the Internet of Things. 2016 [cited 2016 25-01-2016]; Available from: http://thinfilm.no/2015/02/18/smart-labels-will-powerinternet-things/.

[4] Dai, Hong-Ning, et al. "Big data analytics for manufacturing internet of things: opportunities, challenges and enabling technologies." Enterprise Information Systems (2019): 1-25.

[5] Perera, C., et al., Sensing as a service model for smart cities supported by internet of things. Transactions on
Emerging Telecommunications Technologies, 2014. 25(1): p. 81-93.

[6] Sheng, X., et al., Sensing as a service: Challenges, solutions and future directions. Sensors Journal, IEEE, 2013. 13(10): p. 3733-3741.

[7] Liu, C.H., B. Yang, and T. Liu, Efficient naming, addressing and profile services in Internet-of-Things sensory environments. Ad Hoc Networks, 2014. 18: p. 85101.

[8] Avilés-López, E. and J.A. García-Macías, TinySOA: a service-oriented architecture for wireless sensor networks. Service Oriented Computing and Applications, 2009. 3(2): p. $99-108$

[9] Hu, S., et al., AgOnt: Ontology for Agriculture Internet of Things, in Computer and Computing Technologies in Agriculture IV. 2011, Springer. p. 131-137.

[10] Yan-e, D. Design of intelligent agriculture management information system based on IoT. in Intelligent Computation Technology and Automation (ICICTA), 2011 International Conference on. 2011. IEEE.

[11] TongKe, F., Smart Agriculture Based on Cloud Computing and IOT. Journal of Convergence Information Technology, 2013. 8(2).

[12] Liang, Y., et al., Study on the framework system of digital agriculture. Chinese Geographical Science, 2003. 13(1): p. 15-19.

[13] Consortium, O., Open source solution for the internet of things into the cloud, January 2012.

[14] Phenonet. Commonwealth Scientific and Industrial Research Organisation (CSIRO), Australia. 2014 01-092015]; Available from: http://phenonet.com.

[15] Zhao, J.-c., et al. The study and application of the IOT technology in agriculture. in Computer Science and Information Technology (ICCSIT), 2010 3rd IEEE International Conference on. 2010. IEEE.

[16] Xu, L.D., Information architecture for supply chain quality management. International Journal of Production Research, 2011. 49(1): p. 183-198.

[17] Melo, M.T., S. Nickel, and F. Saldanha-da-Gama, Facility location and supply chain management-A review. European journal of operational research, 2009. 196(2): p. 401-412.

[18] Liu, J., S. Zhang, and J. Hu, A case study of an interenterprise workflow-supported supply chain management system. Information \& Management, 2005. 42(3): p. 441454.

[19] Croxton, K.L., et al., The supply chain management processes. The International Journal of Logistics Management, 2001. 12(2): p. 13-36.

[20] Van der Vorst, J.G., S.-O. Tromp, and D.-J.v.d. Zee, Simulation modelling for food supply chain redesign; integrated decision making on product quality, sustainability and logistics. International Journal of Production Research, 2009. 47(23): p. 6611-6631.

[21] Xiaorong, Z., et al., The Design of the Internet of Things Solution for Food Supply Chain. 2015.

[22] Kelepouris, T., K. Pramatari, and G. Doukidis, RFIDenabled traceabilityin the food supply chain. Industrial Management \& Data Systems, 2007. 107(2): p. 183-200.

[23] Barchetti, U., et al. RFID, EPC and B2B convergence towards an item level traceability in the pharmaceutical supply chain. in RFID-Technology and Applications (RFID-TA), 2010 IEEE International Conference on. 2010 IEEE.

[24] Yu, X., et al., Pharmaceutical supply chain in China: current issues and implications for health system reform. Health Policy, 2010. 97(1): p. 8-15. 
[25] Yu, X., F. Sun, and X. Cheng. Intelligent urban traffic management system based on cloud computing and Internet of Things. in Computer Science \& Service System (CSSS), 2012 International Conference on. 2012. IEEE.

[26] Iyyappan, M.S. and M.V. Nandagopal, Automatic Accident Detection and Ambulance Rescue with Intelligent Traffic Light System. International Journal of Advanced Research in Electrical, Electronics and Instrumentation Engineering, 2013. 2(4).

[27] Papazoglou, M.P., et al., Service-oriented computing: State of the art and research challenges. Computer, 2007(11): p. 38-45.

[28] Issarny, V., et al., Service-oriented middleware for the future internet: state of the art and research directions. Journal of Internet Services and Applications, 2011. 2(1): p. 23-45.

[29] $\mathrm{Da} \mathrm{Xu}, \mathrm{L} ., \mathrm{W}$. He, and $\mathrm{S}$. $\mathrm{Li}$, Internet of things in industries: a survey. Industrial Informatics, IEEE Transactions on, 2014. 10(4): p. 2233-2243.

[30] Leguay, J., et al. Service oriented architecture for heterogeneous and dynamic sensor networks. in Proceedings of the second international conference on Distributed event-based systems. 2008. ACM.

[31] Jia, X., et al. RFID technology and its applications in Internet of Things (IoT). in Consumer Electronics, Communications and Networks (CECNet), 2012 2nd International Conference on. 2012. IEEE.

[32] Domingo, M.C., An overview of the Internet of Things for people with disabilities. Journal of Network and Computer Applications, 2012. 35(2): p. 584-596.

[33] Zhiliang, W., et al. A SOA based IOT communication middleware. in Mechatronic Science, Electric Engineering and Computer (MEC), 2011 International Conference on. 2011. IEEE.

[34] Wiggins, G.P. and J. McTighe, The understanding by design guide to creating high-quality units. 2011: ASCD.

[35] Golatowski, F., et al. Service-oriented software architecture for sensor networks. in International Workshop on Mobile Computing, IMC. 2003.

[36] Delicato, F., et al. A flexible web service based architecture for sensor networks. in IEEE Workshop on Mobile and Wireless Networks (MWN 2003), Rhode Island, NY, USA. 2003.

[37] Delicato, F.C., et al. Reflective middleware for wireless sensor networks. in Proceedings of the 2005 ACM symposium on Applied computing. 2005. ACM.

[38] Jang, W.-S., W.M. Healy, and M.J. Skibniewski, Wireless sensor networks as part of a web-based building environmental monitoring system. Automation in Construction, 2008. 17(6): p. 729-736.

[39] Ibbotson, J., et al. Sensors as a service oriented architecture: Middleware for sensor networks. in Intelligent Environments (IE), 2010 Sixth International Conference on. 2010. IEEE.

[40] Decker, G., et al. BPEL4Chor: Extending BPEL for modeling choreographies. in Web Services, 2007. ICWS 2007. IEEE International Conference on. 2007. IEEE.

[41] Lanese, L., et al. Bridging the gap between interaction-and process-oriented choreographies. in Software Engineering and Formal Methods, 2008. SEFM'08. Sixth IEEE International Conference on. 2008. IEEE.

[42] Sundmaeker, H., et al., Vision and challenges for realising the Internet of Things. Cluster of European Research Projects on the Internet of Things - CERP IoT, 2010.

[43] Srinivasan, C. R., et al. "A review on the different types of Internet of Things (IoT)." Journal of Advanced Research in Dynamical and Control Systems 11.1 (2019): 154-158.

\section{Authors' Profiles}

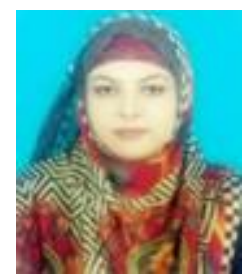

Computing

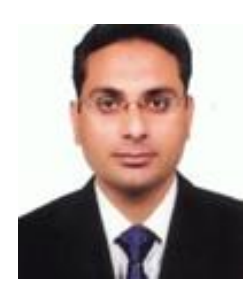

Dr. Muhammad Asif has received his $\mathrm{PhD}$ in Computer Science from the department of computer and information science (IDI), Norwegian University of Science and Technology (NTNU), Norway. Currently, he is working as an Associate Professor in Department of Computer Science, University of Lahore, Pakistan. His research interests are Internet of Things, Mobile Information Systems and Data Sciences.

How to cite this paper: Maha Arooj, Muhammad Asif, " Sensing as a Service: Vision, Practices and Architecture", International Journal of Information Engineering and Electronic Business(IJIEEB), Vol.11, No.6, pp. 37-43, 2019. DOI: 10.5815/ijieeb.2019.06.06 